\title{
Loophole-Free Test of Einstein-Podolsky-Rosen Steering with One Bit of Faster-than-Light Communication
}

\author{
M. D. Mazurek, ${ }^{1,2}$ Y. Xiang, ${ }^{3}$ M. J. Stevens, ${ }^{1}$ J. C. Bienfang, ${ }^{4,5}$ M. A. Wayne, ${ }^{5}$ C. \\ Abellán, ${ }^{6}$ W. Amaya, ${ }^{6}$ M. W. Mitchell, ${ }^{6,7}$ R. P. Mirin, ${ }^{1}$ S. W. Nam, ${ }^{1}$ Q. He, ${ }^{3}$ L. K. \\ Shalm, ${ }^{1,2}$ and H. M. Wiseman ${ }^{8}$ \\ ${ }^{l}$ NIST-National Institute of Standards and Technology, 325 Broadway, Boulder, Colorado 80305, USA; \\ ${ }^{2}$ Department of Physics, 390 UCB, University of Colorado, Boulder, Colorado 80309, USA; ${ }^{3}$ State Key \\ Laboratory for Mesoscopic Physics, School of Physics, Nano-optoelectronics Frontier Center of the Ministry of \\ Education \& Collaborative Innovation Center of Quantum Matter, Peking University, Beijing 100871, China; \\ ${ }^{4}$ Joint Quantum Institute: University of Maryland and NIST, 100 Bureau Drive, Gaithersburg MD 20899, USA; \\ ${ }^{5}$ NIST, 100 Bureau Drive, Gaithersburg MD 20899, USA; ${ }^{6}$ ICFO-Institut de Ciencies Fotoniques, The Barcelona \\ Institute of Science and Technology, 08860 Castelldefels (Barcelona), Spain; ${ }^{7}$ ICREA-Institució Catalana de \\ Recerca i Estudis Avançats, 08010 Barcelona, Spain; ${ }^{8}$ Centre for Quantum Computation and Communication \\ Technology (Australian Research Council) \& Centre for Quantum Dynamics, Griffith University, Brisbane, \\ Queensland 4111, Australia \\ michael.mazurek@nist.gov
}

\begin{abstract}
The communication cost for classically simulating Einstein-Podolsky-Rosen (EPR) steering correlations quantifies their strength. We report a loophole-free demonstration of EPR steering correlations requiring more than one faster-than-light bit to simulate. (C) 2020 The Author(s)
\end{abstract}

OCIS codes: (270.0270) Quantum optics; (270.5585) Quantum information and processing

\section{Introduction}

In 1935, Einstein, Podolsky and Rosen (EPR) put forward their famous paradox to declare the quantum mechanical description of physical reality incomplete [1]. Their argument relied on the remarkable feature of bipartite quantum entanglement, that the choice of measurement by one party (Alice) seems to affect, instantaneously, the type of state held by the second party (Bob). In the same year Schrödinger called the phenomenon "steering" [2], and discussed the possibility of using arbitrarily many types of measurement. More recently, Schrödinger's generalization was formalized as EPR steering: the experimental violation of the asymmetric model comprising a local hidden state quantum model for Bob's system and a local hidden variable model to generate Alice's measurement results [3]. From the perspective of Schrödinger, who was convinced of the completeness and correctness of the quantum state as a description of a local system, EPR steering implied a genuinely superluminal effect [2]. This raises the foundational question of how much faster-than-light (FTL) classical communication would be required for Alice to steer Bob's state, in the absence of entanglement. It has been shown theoretically that an infinite amount of classical communication from (untrusted) Alice to (trusted) Bob is necessary to simulate all EPRsteering correlations for any pure entangled two-qubit state $[4,5]$. For any experimentally accessible correlations, the required amount of classical communication will be finite, and moreover is a measure of the strength of EPR steering. Here we report on the first demonstration of EPR-steering correlations that require more than one bit of FTL communication to simulate classically, in a loophole-free experiment.

\section{Methods and Results}

We consider the scenario in which Bob has a particle on which he can perform a measurement in one of three pre-determined bases, and after learning Bob's measurement setting, Alice tries to guess his outcome. We assume that Bob's particle is modelled as a local hidden state, and that all the equipment in Bob's lab is trusted, wellcharacterized, and operates according to local quantum mechanics. In the theory we allow Alice access to a single bit of information about Bob's measurement choice, which she can use — in a faster-than-light way — to influence Bob's particle and thus his measurement outcome. Under this specific set of assumptions we derive a linear steering inequality that represents an upper bound on the extent to which Alice can steer Bob's state.

The inequality can in principle be violated if, in each experimental trial, Alice has access to more than one FTL bit, or (more realistically) if she can perform her own measurement on a particle that is entangled with Bob's. It is convenient to invoke a third party Charlie who prepares an entangled pair of qubits and distributes it to Alice and Bob. Ideally the pair is prepared in the maximally entangled singlet state, and Alice and Bob each have the 


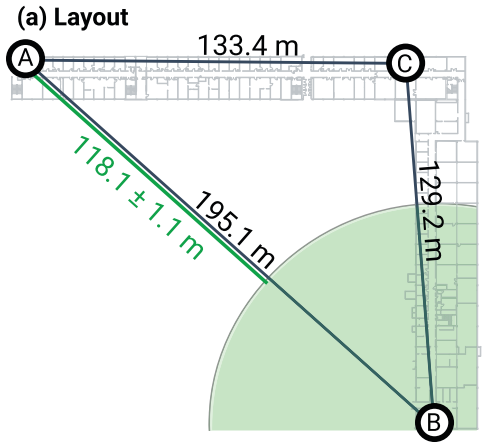

(b) Alice/Bob

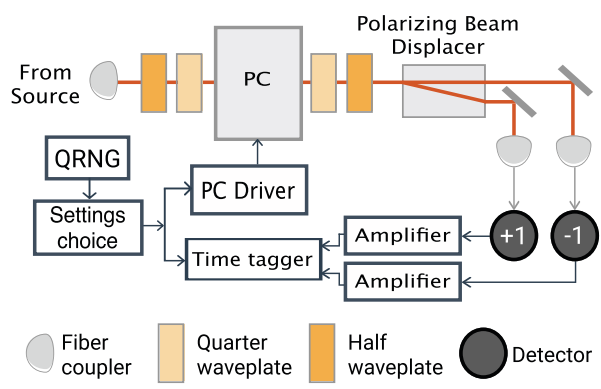

(c) Bob's measurement settings

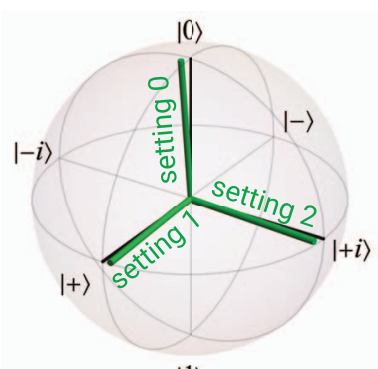

$|1\rangle$

Fig. 1: (a) Charlie (C) produces entangled photon pairs and distributes them to Alice (A) and Bob (B). The moment Alice records her measurement outcome, information about Bob's measurement setting choice cannot have propagated outside the green light-cone emanating from Bob's lab. (b) Alice's and Bob's measurement stations. A quantum random number generator (QRNG) chooses one of three measurement settings, which is implemented via fixed waveplates, a single Pockels cell (PC), a polarizing beam displacer, and two detectors, labelled with +1 and -1 outcomes. (c) Bloch sphere representation of Bob's target (black lines) and actual (green cylinders) measurement bases. The vectors represent the +1 eigenstates of each basis. The radii of the green cylinders represent the 5-sigma uncertainty on Bob's implemented measurements.

ability to perform projective measurements in the same set of three mutually unbiased bases. We only consider trials in which Alice and Bob perform their measurements in the same basis, in which case their outcomes will be anti-correlated and Alice will be able to correctly predict Bob's measurement outcome and thus maximally violate the inequality.

Due to the asymmetric nature of the EPR-steering scenarios, the requirements for performing a LHF experiment are asymmetric as well. We consider three well-recognized loopholes; we require Alice to report her measurement outcome before she can learn information about Bob's measurement setting (closing the locality loophole), we require Alice to report an outcome for every trial of the experiment (closing the efficiency loophole), and we require Alice and Bob's settings to be chosen randomly and independently from each other (closing the freedomof-choice loophole).

Our experimental setup is displayed in Fig. 1. Charlie uses a polarization-encoded photon-pair source [6] to produce pairs of photons in the maximally entangled singlet state (Fig. 1(a)). He sends one photon to Alice's lab, and the other to Bob's. Alice and Bob then each use a QRNG and a Pockels cell to rapidly choose and switch between measurement settings (Fig. 1(b)). The overall system detection efficiency for Alice's +1 and -1 outcomes are $75.1 \pm 0.4 \%$ and $73.9 \pm 0.3 \%$, respectively. The bound of the inequality depends on the actual measurements Bob implements, which we characterize via quantum measurement tomography. Fig. 1(c) shows that Bob was able to quickly switch between three measurement settings that were sufficiently close to the three ideal settings. We perform $2.8 \times 10^{6}$ trials of the experiment, and violate our inequality by 36 standard deviations.

Our results are the first to demonstrate EPR-steering correlations that require more than one FTL bit to simulate classically, paving the way for future experiments with a greater number of measurement settings that can verify even stronger EPR steering.

\section{References}

1. A. Einstein, B. Podolsky, and N. Rosen, "Can quantum-mechanical description of physical reality be considered complete?” Phys. Rev. 47, 777-780 (1935).

2. E. Schrödinger, "Discussion of probability relations between separated systems," Math. Proc. Camb. Philos. Soc. 31, 555-563 (1935).

3. H. M. Wiseman, S. J. Jones, and A. C. Doherty, "Steering, entanglement, nonlocality, and the EinsteinPodolsky-Rosen paradox," Phys. Rev. Lett. 98, 140402 (2007).

4. S. Nagy and T. Vértesi, "EPR steering inequalities with communication assistance," Sci. Reports 6, 21634 (2016).

5. A. B. Sainz, L. Aolita, N. Brunner, R. Gallego, and P. Skrzypczyk, "Classical communication cost of quantum steering," Phys. Rev. A 94, 012308 (2016).

6. L. K. Shalm, E. Meyer-Scott, B. G. Christensen, P. Bierhorst, M. A. Wayne, et al., "Strong loophole-free test of local realism," Phys. Rev. Lett. 115, 250402 (2015). 